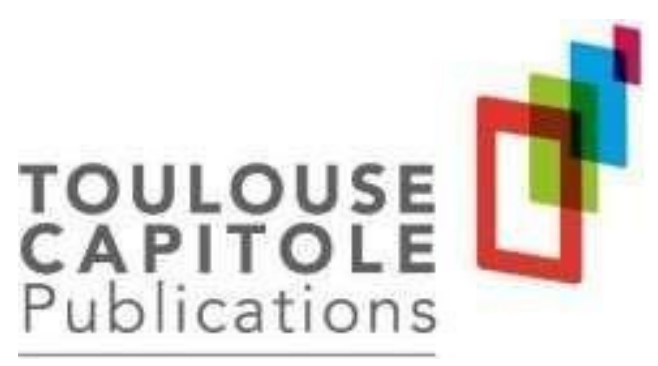

« Toulouse Capitole Publications » est l'archive institutionnelle de l'Université Toulouse 1 Capitole.

Désobéir pour être : les Noirs américains

MASTOR WANDA

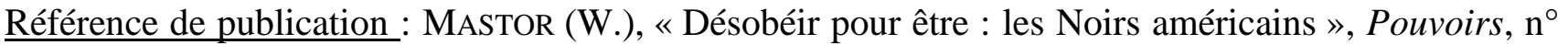
155,2015 , p. 81-95.

Pour toute question sur Toulouse Capitole Publications, contacter portail- publi@ut-capitole.fr 


\section{Désobéir pour être : les Noirs américains}

« La société de bus RTA rend hommage à la femme qui s'est tenue debout en restant assise. », Inscription accompagnant la photo de Rosa Parks au moment de ses funérailles sur les premières places du bus de Montgomery (2 novembre 2005)

Le 15 septembre 1963, quatre enfants noirs sont tués lors d'un attentat à la bombe contre l'église baptiste de Birmingham en Alabama. Les individus interpellés à l'époque, membres du Ku Klux Klan, ne furent condamnés qu'à six mois de prison avec sursis. Il faudra attendre près de quarante années pour que l'un des quatre hommes auteurs du crime raciste soit condamné à la perpétuité.

Les États-Unis n'en finissent pas d'expier ce passé raciste et ségrégationniste, d'exprimer régulièrement une repentance à l'égard d'une population humiliée et maltraitée - à l'image de ce bus de Montgomery dont l'entreprise rend, en 2005, hommage à l'une des icônes du mouvement en faveur des droits civiques et qui, dans les années 1950, l'interdisait de s'asseoir à l'avant. Mais la douleur et les souffrances ne s'atténuent guère et il est malheureusement impossible d'affirmer qu'elles appartiennent au passé.

Le 17 juin 2015, un tireur abat neuf personnes dans une église de Charleston en prononçant la sentence suivante : «Vous violez nos femmes et envahissez notre pays. Vous devez partir. 》 De nombreuses années se sont écoulées entre ces deux attentats qui ont pour point commun d'avoir touché des églises emblématiques de la communauté noire. Mais ce ne sont pas des actes isolés - ni perpétrés par des « déséquilibrés »-, et la liste des meurtres racistes est d'autant plus terrifiante qu'elle ne contient pas les actes ordinaires. De la révolte du ghetto de Watts à Los Angeles en 1965 à celles médiatiques de Ferguson en 2014, en passant par les émeutes raciales de Newark, de Detroit en 1967, les suites meurtrières de l'assassinat de Martin Luther King, les violences dans le quartier de Liberty City à Miami en 1980, celles engendrées par les coups portés à Rodney King en 1992 et l'assassinat de Timothy Thomas en 2001 par des policiers, 1'histoire des États-Unis peut aussi être racontée à travers l'opposition des deux races - opposition dont les reflets irriguent tous les champs de la vie en société : le droit, l'économie, la sociologie, et dont les prolongements artistiques (musique, littérature, cinéma, peinture) sont particulièrement remarquables. Le premier Noir titulaire d'un doctorat à Harvard, William Edward Burghardt Du Bois, l'avait déjà énoncé en ouverture de sa remarquable œuvre sur Les Âmes du peuple noir : «Le problème du xxe siècle est le problème de la ligne de partage des couleurs 1. » Sans doute savait-il, tant son œuvre lyrique est porteuse d'un espoir que le lecteur sent lointain, qu'il serait aussi celui du xxie siècle. Assurément, le problème est actuel et on pourrait nous rétorquer qu'il n'est pas spécifiquement américain. La haine de l'autre est universelle mais reste une particularité des États-Unis compte tenu de l'histoire de la présence des Noirs sur leur sol. Comme l'ont démontré Lauren Robel et Élisabeth Zoller, « de la guerre fratricide qui l'ensanglanta au xixe siècle à la lutte pour la justice raciale qui menace de la déchirer demain, l'Amérique n'en finit pas d'expier le péché originel qui souilla sa création, l'esclavage 2 ». Certains préjugés trouvent une assise juridique, même constitutionnelle, d'autres sont latents, ordinaires, non officiels donc incontrôlables et emportent la même conséquence. Celle de l'exclusion. Et « le préjugé antinoir, écrit Martin Luther King, fait partie intégrante de la personnalité 
américaine; ce préjugé s'est nourri de la doctrine de l'infériorité d'une race par rapport à l'autre 3 ». Car l'histoire de la ligne de partage des couleurs a toujours, sans exception, été celle de la domination d'une race sur l'autre. Domination du maître sur l'esclave, du Blanc sur le Noir, depuis les normes les plus suprêmes jusqu'aux actes les plus ordinaires : boire à une fontaine, se laver les mains, entrer dans une cabine d'essayage, s'accouder à un comptoir, aller à l'école, s'asseoir dans un bus. La théorie de la séparation des races était perverse en ce qu'elle était légitimée par ses promoteurs pour son caractère non excluant : la ségrégation n'a pas pour effet de placer une race dans une situation d'infériorité ; elle ne fait que séparer.

$\mathrm{Si}$ on ajoute à cette histoire de l'esclavage et de la ségrégation le fait que le peuple américain a porté au plus haut niveau du pouvoir un Noir4, on obtient un espace de réflexion exceptionnel sur l'opposition des races.

Pour étudier la question de la désobéissance civile des Noirs, ayons sans cesse à l'esprit que l'ampleur des émeutes raciales aujourd'hui, la permanence de la ségrégation de facto et l'impuissance des autorités à éradiquer des violences profondément ancrées dans une société où le port d'armes est de surcroît libre ne peuvent s'appréhender abstraction faite du mal originaire. L'ère de l'esclavage, marquée par la supériorité de la race blanche sur la race noire, allait céder ensuite la place à l'ère de la séparation. L'esclavage moral succédait à l'esclavage physique, et l'enfermement était d'autant plus violent qu'il sévissait dans les États pendant que les autorités fédérales (Congrès et Cour suprême) allaient dans le sens de l'émancipation. C'est dans cette période que les Noirs, qui, en tant qu'esclaves, trouvaient dans la fuite vers les États libres le seul moyen de désobéir, vont décider de combattre : « Esclaves héréditaires ! Ne savez-vous pas que celui qui veut se libérer doit se battre 5 ? » Menés par l'énergie hors du commun de la National Association for the Advancement of Colored People et de Martin Luther King, ils vont choisir la voie de la résistance pacifique : former systématiquement des recours contre les lois ségrégationnistes des États, refuser de céder leur place dans un bus, aller dans des écoles réservées aux Blancs, organiser des marches pacifistes malgré les interdictions de manifester. «En se révoltant, les Noirs n'attaquèrent pas seulement la cause extérieure de leur misère : leur révolution les révéla à eux-mêmes. Le Noir était quelqu'un. Il découvrait le sens de sa personnalité et il lui fallait, très vite, se libérer 6. » Indispensable, la désobéissance ne fut pas suffisante. À l'enchantement de la déségrégation de droit succède vite le désenchantement de la ségrégation de fait. Les Noirs ont alors compris et accepté qu'ils ne pourraient accéder au statut d'hommes libres (c'est- à-dire placés dans des conditions de vie strictement identiques à celles des Blancs) qu'à la condition de forcer leur intégration. S'ouvrira alors l'ère des mal nommées discriminations positives, que Martin Luther King avait déjà appelée de ses vœux.

\section{Naître pour obéir}

Au commencement, le Noir américain était esclave. Un bien destiné à servir et obéir. Telle est l'hérédité des Afro-Américains que ne peuvent occulter les débats contemporains sur le racisme et les moyens d'y remédier. Tous les apôtres de la révolution non violente et les défenseurs des droits civiques ne cessent de rappeler cette inextricable spécificité du Noir américain : " être Noir, en Amérique, c'est porter la trace historique de l'esclavage et du démembrement familial 7. » La question de l'esclavage a marqué les travaux de la Convention de Philadelphie du sceau de la contradiction ; certains grands fondateurs de la patrie américaine possédaient des esclaves et coexistaient, dans la Constitution de 1787, la volonté de « former une union plus parfaite », d'y " établir la justice », et la clause des esclaves fugitifs : «Nul individu tenu au service ou au travail dans un État conformément à ses lois, et qui viendrait à 
s'échapper dans un autre, ne sera déchargé de ce service ou de ce travail en vertu des lois de ce dernier, mais il devra être rendu sur simple requête de la partie à laquelle ce service ou ce travail pourra être dû » (art. IV, sect. 2, al. 3). Lincoln lui-même avait envisagé comme solution salvatrice de l'Union l'hypothèse du rapatriement des esclaves dans leur continent d'origine 8, notamment dans un État spécialement créé à cet effet, le Liberia.

Dans une certaine mesure, les arrêts de la Cour suprême sur la ségrégation ont été «préparés » par ceux sur l'esclavage. Dès l'arrêt Prigg v. Pennsylvania de 18429, le juge Roger Brooke Taney développe dans une opinion concordante les idées qui deviendront celles de la majorité dans l'arrêt Dred Scott $v$. Sandford de 185710. Déclarant le Fugitive Slave Act de 1793, qui permettait à un propriétaire d'esclaves de capturer des fugitifs qui auraient rejoint un État abolitionniste, constitutionnel, le juge Taney insiste au-delà sur l'obligation pour tous les États d'aider les propriétaires d'esclaves à capturer leurs fugitifs. Le juge concordant avait déjà défendu avec véhémence l'institution de l'esclavage alors qu'il était Attorney General sous l'administration Jackson, déclarant à son Président que « la race africaine aux États-Unis, même si elle est libre, constitue une classe inférieure, et n'exerce aucune influence poli- tique. [...] C'est la seule catégorie de personnes susceptible d'être la propriété de quelqu'un. [...] Elles n'ont jamais été considérées comme une portion de la souveraineté d'aucun État et, en conséquence, ne sont pas concernées par le terme citoyens $11 »$. Rédigeant l'opinion de la Cour dans 1'affaire Dred Scott v. Sandford, le juge Taney offrira même à l'esclavage un statut constitutionnel : "Le droit de propriété sur un esclave est clairement et expressément affirmé dans la Constitution. » Hommage doit être rendu aux juges John McLean et Benjamin Curtis. Sans doute n'imaginaient-ils pas à l'époque que l'affirmation dans leurs opinions dissidentes de la possibilité de citoyenneté d'un Noir allait devenir, dix ans plus tard, l'objet du remaniement de la Constitution. L'opinion dissidente du premier est une condamnation morale de l'esclavage ; celle du second, plus incisive, condamne l'interprétation de la Constitution par la majorité et développe la conception de la double citoyenneté, consacrée en 1868 par le XIVe amendement.

« La question qui nous est posée, développe la Cour, est de savoir si les personnes visées dans la fin de non-recevoir font partie de ce peuple et si elles sont un élément constitutif de cette souveraineté. Nous pensons qu'elles ne le sont pas, qu'elles ne sont pas comprises dans cette souveraineté, qu'on n'a jamais voulu les inclure dans le groupe des « citoyens » visés dans la Constitution et qu'elles ne peuvent donc revendiquer aucun des droits et privilèges que cet instrument accorde et garantit aux citoyens des États-Unis. Bien au contraire, on les considéra à l'époque comme un groupe d'individus subordonnés et inférieurs qui avaient été assujettis par la race dominante et qui, affranchis ou non, demeuraient sujets à l'autorité de cette dernière et qui n'avaient d'autres droits ou privilèges que ceux que les gouvernants et le gouvernement pouvaient choisir de leur accorder 12. " La Cour poursuit en indiquant qu'il ne lui appartient pas d'émettre un jugement de valeur, que sa compétence se limite à l'application de la Constitution et à son interprétation conformément notamment aux intentions des constituants. À leur propos, elle rappelle qu'ils ne faisaient alors que partager une opinion communément admise : «Pendant plus d'un siècle, [les esclaves] ont été considérés comme des êtres inférieurs, inaptes de manière générale à s'associer avec la race blanche, que ce soit dans les relations sociales ou politiques, considérablement si inférieurs qu'ils ne détenaient aucun droit qu'un homme blanc fût tenu de respecter, de telle sorte que le nègre pouvait justement et légitimement être réduit en esclavage pour son avantage. Il fut acheté, vendu et traité comme une marchandise ordinaire et un objet de commerce, chaque fois qu'un profit pouvait en résulter. À l'époque, cette opinion était établie et universelle dans la partie civilisée de la race blanche. On la tenait comme un axiome moral et politique que personne ne songeait à contester ou considérer comme sujet à controverse 13. » 
La littérature raciste relative aux Noirs fut donc également, et souvent, œuvre des autorités créatrices du droit. Des hommes politiques aussi peu controversés que Thomas Jefferson ont pu écrire des pages renforçant la Cour suprême dans son attitude esclavagiste puis ségrégationniste. Il est ainsi possible de lire dans ses Notes sur l'État de Virginie de 1785 que, « au-delà de la couleur, des traits et des cheveux, il existe d'autres éléments physiques distinctifs prouvant la différence des races. [Les Noirs] ont une pilosité moins importante. Ils sécrètent moins par les reins que par les glandes de la peau, ce qui entraîne une odeur forte et désagréable. [...] Ils sont plus ardents avec leurs femmes: mais l'amour semble être pour eux plus l'expression d'un brûlant désir qu'un mélange de sentiment et sensation. [...] En général, leur existence semble être gouvernée plus par l'instinct que par la réflexion 14 ».

Le XIIIe amendement abolit l'esclavage physique mais ne libéra pas les Noirs. L'Union avait survécu à la guerre civile mais le conflit des races ne faisait que commencer. Malgré les avancées formelles, le Noir passant en quelques années du statut d'objet à celui de citoyen, la réalité offrait le spectacle d'une race qui, effectivement, était toujours captive. Des ghettos, des inégalités, de la pauvreté, de la honte d'être ou plutôt de n'être pas. «Il y a au moins une chose que tous les hommes savent, écrit Du Bois, malgré les compromis, la guerre et les combats, le Noir n'est pas libre. [...] Payer des impôts, et ne pas être représentés: telle est la règle de leur vie politique 15. » Le réveil des âmes noires se fit en même temps que l'adhésion à une méthode. Ce sera celle de la révolution non violente, de la désobéissance civile.

\section{Désobéir pour survivre}

La sortie de l'ère de l'esclavagisme a permis de tourner la page de l'aveu de l'infériorité d'une race; celle de la ségrégation, sous couvert d'une simple séparation des races, ne signifiait en réalité pas autre chose. Il est classique de présenter l'arrêt Brown v. Board of Education of Topeka de 195416 comme mettant fin à la ségrégation raciale, mais les choses sont en réalité plus complexes: nombreux sont les arrêts de la Cour suprême et des cours fédérales sur la question de la ségrégation qui ont permis des avancées mais aussi de nets reculs. Les deux mouvements étant en réalité étroitement liés à la question de la concurrence des compétences de l'Union et des États 17. Il ne faut pas oublier que, pendant que la ségrégation sévissait dans les États, le Congrès fédéral votait plusieurs lois visant à intégrer les Noirs dans la société civile et politique : la loi sur les droits civils de 1866, l'adoption des XIIIe, XIVe et XVe amendements entre 1865 et 1869, la troisième loi sur les droits civils de 1875 interdisant la discrimination raciale « dans les lieux ouverts à l'usage du public, y compris les auberges, les lieux d'accommodements du public, les théâtres, et tous les lieux publics de divertissement ». Les États du Sud, bénéficiant de la complicité de la Cour suprême, allaient violemment réagir à cet arsenal libertaire en estimant que le Congrès n'avait pas le pouvoir d'interdire la ségrégation. De l'avis du pouvoir judiciaire, il appartenait donc aux États et non à l'Union de décider du sort des Noirs.

Les conséquences de l'arrêt Brown de 1954 ne furent cependant pas aussi spectaculaires que le revirement de la Cour. Cette dernière précise que la question de l'enseignement public ne se pose pas dans les mêmes termes en 1954 qu'en 1868 (date de l'adoption du XIVe amendement) et au jour de la décision, où « nombreux sont les Noirs qui rencontrent un succès éclatant dans le domaine des arts et des sciences, mais aussi dans les affaires et le monde professionnel $18 »$. Ce qui justifie à ses yeux l'inutilité de faire « remonter l'horloge à 1868, date à laquelle l'amendement fut adopté, ou même à 1896, lorsque fut rendu l'arrêt Plessy v. Ferguson 19 ». La Cour, sous la plume de son président Warren, conclut que, « dans le 
domaine de l'enseignement public, la doctrine « séparés mais égaux » n'a pas sa place 20 ». Par bien des aspects, l'arrêt Brown est un grand arrêt. Mais il n'a pas réussi à éradiquer la ségrégation raciale de facto. Comme l'affirmait la Cour dans l'arrêt Plessy de 1896 21, « le droit est impuissant à éradiquer les instincts raciaux et à abolir les distinctions fondées sur des différences physiques 22 »; l'élection d'un président noir, aussi puissant que soit le symbole, n'a pas entraîné le règne subit de la mixité. Dans son discours sur la question raciale du 24 mars 2008, Barack Obama reconnaît lui-même que « la ségrégation à l'école a produit et produit encore des écoles inférieures. Cinquante ans après l'arrêt Brown v. Board of Education, rien n'a changé et la qualité inférieure de l'éducation que dispensent ces écoles aide à expliquer les écarts de réussite entre les étudiants blancs et noirs aujourd'hui». Les émeutes raciales de Ferguson et de Baltimore qui ont éclaté pendant son second mandat ont fatalement renforcé son pessimisme vis-à-vis de la réalité de l'égalité des races.

Ce qui est particulièrement remarquable dans l'histoire des Afro- Américains, c'est la réaction de ce peuple face à des siècles d'humiliation dégradante. Certains Blancs ont bien évidemment très tôt condamné l'esclavage, à l'image de Henry David Thoreau, à qui il est classique d'attribuer l'une des paternités de la notion de désobéissance civile. «Quel est le gouvernement qui s'impose à un homme face à ce gouvernement américain, aujourd'hui ? Je réponds qu'il ne peut sans honte y être associé. Je ne puis un seul instant reconnaître cette organisation poli- tique pour mon gouvernement puisqu'elle est aussi le gouvernement de l'esclave 23. » Thoreau avait refusé de payer une taxe destinée à financer la guerre contre le Mexique et exhortait ses compatriotes à employer ce moyen de résistance non violente : «Si un millier d'hommes refusaient de payer leurs impôts cette année, ce ne serait pas une mesure violente et sanguinaire, comme le fait de les payer et permettre par là à l'État de commettre la violence et de verser le sang innocent. Telle est, en fait, la définition d'une révolution paisible, si semblable chose est possible 24. » Propos qui ne sont pas sans rappeler ceux de La Boétie : «Or ce tyran seul, il n'est pas besoin de le combattre, ni de l'abattre. Il est défait de lui-même, pourvu que le pays ne consente point à sa servitude. Il ne s'agit pas de lui ôter quelque chose, mais de ne rien lui donner 25. »

La désobéissance pacifiste prônée par Martin Luther King était d'autant plus admirable que les actes racistes meurtriers ou ordinaires étaient d'une violence inimaginable. La lecture de l'ensemble de l'œuvre du pasteur noir, à aucun moment porteuse d'un message de haine envers les Blancs, est troublante de dignité. Le ton est donné dès la dédicace de Où allons-nous ? 26, qui s'adresse tant aux Noirs qu'aux Blancs qui luttent en faveur des droits civiques. Bien loin des discours de Malcolm X (à l'image de son dernier prononcé quelques jours avant son assassinat: "Nous croyons que les pratiques mauvaises à l'encontre des Noirs dans cette société sont criminelles et que ceux qui engagent de telles pratiques criminelles ne sont rien d'autre que des criminels. Et nous estimons être en droit de nous battre contre ces criminels, par tous les moyens nécessaires »), Martin Luther King croit, lui, à la révolution pacifiste, comme en son temps Du Bois: «Par tous les moyens civilisés et non violents possibles, nous devons lutter pour les droits que le monde accorde aux hommes [... 27. »

Le but de la désobéissance n'est pas seulement d'extraire le Noir de son statut d'être inférieur mais également de lui permettre d'accéder aux mêmes droits que ceux des Blancs. Et pour parvenir à cette mission semblant, au début des années 1960, étrangère au monde des possibles, le fils, petit-fils et arrièrepetit-fils de prédicateurs puise dans la foi les armes du combat qui ne saurait être autre que pacifiste. La référence à Dieu est omniprésente dans les discours, textes et lettres, et c'est en toute logique et cohérence que Martin Luther King est animé de la passion de la paix. Ces armes, ce seront le boycott des bus, les occupations des comptoirs et des églises réservés aux Blancs, les marches sur les villes minutieusement 
choisies. Malgré les attaques physiques, les quarante mille Noirs de Montgomery marchent en 1955 pendant des vingtaines de kilomètres pour aller travailler. Les Noirs menés par le pasteur marchent sur Albany (1961 et 1962), Birmingham (1963), Washington (1963), Selma (1964), Chicago (1966). La foule est immense et les espoirs ne se limitent pas aux discours du futur prix Nobel de la paix ; en 1964 et 1965 seront votés le Civil Rights Act et le Voting Rights Act. Les objectifs, méthodes et déroulements de ce programme de lutte pour les droits civiques jalonnent l'œuvre héroïque de Martin Luther King, trop souvent réduite à quelques discours. L'expression « désobéissance civile » est explicitement employée lorsque le leader du mouvement pour la défense des droits civiques décrit la révolution noire de l'été 1963. À Birmingham, bravant l'interdiction de manifester, King affirme : « Je voulais être l'un des premiers à donner l'exemple de la désobéissance civile »- une désobéissance étrangère à la force, puissante et juste, qui « frappe sans blesser et ennoblit celui qui la brandit » 28. Une désobéissance aux fondements religieux et philosophiques - il est juste de désobéir à une loi injuste - et aux actes parant leurs auteurs du drap de la dignité, eux qui vivaient jusque-là dans le voile 29 : la maîtrise de soi, face aux violences physiques et morales, requiert une volonté, un courage et une dignité supérieurs au réflexe des coups. Peu avant son assassinat, Martin Luther King réaffirmait que « l'action non violente de masse reste l'une des tactiques les plus efficaces du mouvement pour la liberté », toujours animé du " devoir d'être contre tout ce qui veut obtenir notre liberté par les moyens qui caractérisent nos oppresseurs : la malhonnêteté, la haine, la violence » 30. Désobéissance de masse qui n'en demeurait pas moins soigneusement organisée : pour ses promoteurs, quatre étapes fondamentales devaient la caractériser : l'examen des faits permettant de constater l'injustice ; la négociation ; «l'examen de conscience »; et « l'action directe » 31. Tout était minutieusement prévu : les lieux des manifestations, les ressources financières destinées à payer les cautions des manifestants enfermés, l'appel aux services juridiques de la National Association for the Advancement of Colored People et au soutien de la Southern Christian Leadership Conference. Les mouvements de désobéissance des Noirs dans les années 1960 n'étaient pas une révolte spontanée mais une révolution organisée.

L'œuvre de cette révolution noire fut donc nécessaire mais insuffisante. Nécessaire pour que le Noir ne soit plus qu'une simple couleur indigne, mais insuffisante pour le hisser au même niveau que les Blancs. Et au-delà des effets concrets des avancées juridiques et politiques subsistait la peur : « être noir, en Amérique, c'est l'angoisse et la souffrance d'avoir vu tant de fois l'espoir mourir avant d'être né 32. » Martin Luther King, le pasteur Ralph Abernathy, Edgar Nixon de la National Association for the Advancement of Colored People, pour ne citer qu'eux, avaient déjà réalisé cette fatalité malgré les avancées dont ils avaient été les principaux acteurs. Ils savaient que, pour que l'espoir ne meure plus, il fallait l'aider à s'imposer durablement par le biais d'actions positives contraignantes. Ils savaient qu'il fallait obliger la race toujours dominante à intégrer les Noirs dans leur système de pensée, de vie en société. Les Blancs avaient cru dans un premier temps qu'il « suffisait » à l'homme noir qu'il soit sorti de son statut de chose. Puis ils ont cru que le droit de vote leur serait suffisant, comme ils ont cru des années plus tard que la déségrégation était pour eux une fin en soi. Mais les Noirs voulaient toujours plus, et ce plus n'était rien d'autre que l'effectivité de l'égalité des chances. Les figures emblématiques de la désobéissance civile avaient déjà prédit que seule l'intégration forcée pourrait enfin leur permettre de réaliser cet objectif de toujours : « Aussi navrant que cela puisse paraître, le Noir est désormais convaincu que jamais l'Amérique ne lui accordera l'égalité à moins d'y être forcée 33. »

\section{Forcer l'intégration pour être}

Ne plus être séparés ne signifiait pas vivre ensemble. L’Union avait survécu à la querelle entre le Nord et 
le Sud, elle devait à présent tenter de survivre à la querelle des races. « Une maison divisée contre ellemême court à sa propre perte » : le discours du 16 juin 1858 d'Abraham Lincoln est célèbre et, plus d'un siècle plus tard, Martin Luther King semble lui répondre : «Mais il n'est pas trop tard pour rentrer à la maison 34. » Cette accession des Noirs au rang de citoyens «normaux », placés dans des conditions de stricte égalité avec les Blancs, n'était encore qu'un espoir à l'époque de la révolution noire. Les juges constataient régulièrement la violation du principe d'égalité dans tous les domaines : accès à l'emploi, à l'éducation, à la justice, au logement. Le Noir n'était toujours pas l'égal du Blanc mais, surtout, n'avait pas encore réussi à devenir digne, à croire en ses potentialités. L'ennemi du Noir n'était pas seulement l'autre, c'était aussi lui-même et le renvoi permanent de sa couleur à son passé d'esclave puis d'exclu. Sans doute est-il impossible de mesurer et com- prendre cette plaie égotique : quand on naît marqué du sceau de l'infériorité et qu'enfant on a l'interdiction d'aller dans les écoles pour les Blancs, de jouer dans des parcs réservés aux Blancs, de prier dans les églises blanches et de s'asseoir aux premières places des bus, comment, devenu adulte, peut-on pleinement croire en ses chances mais aussi en ses potentialités ? Et comment, une fois les lieux publics ouverts à tous sans distinction raciale, croire en la possibilité d'une progression indépendamment des Blancs? « N'oublie pas, n’oublie jamais que c'est aux Blancs de décider de ton sort. Peut-être que ce n'est pas bien, mais c'est ainsi. Et tant que tu fréquenteras leur école, tant qu'ils t'enseigneront ce que tu as besoin d'apprendre, tu te conduiras avec soumission, prudence et respect. Parce que tu as la tête dans la gueule du lion 35. » Dans la conquête de leur dignité, les Noirs allaient peu à peu comprendre que non seulement ils pouvaient devenir des êtres indépendamment de l'aide des Blancs, mais qu'ils pouvaient aussi apporter quelque chose à l'Amérique, eux, les anciens esclaves, et passer du statut de serviteur d'un autre à celui de serviteur de la nation. « La riche et amère profondeur de leur expérience, les trésors inconnus de leur vie intérieure, les étranges déchirements de la nature dont ils ont été témoins, pourraient offrir au monde de nouvelles façons de voir 36. »

Nul besoin de revenir ici sur l'historique des politiques d'affirmative action, les controverses qu'elles ont suscitées et qu'elles sus- citent encore 37. Comme pour tant d'autres sujets sophistiqués de la sphère juridique, la doctrine n'échappe pas à l'alternative «pour » ou «contre », et les arguments sont utilisés comme ils doivent l'être dans toute rhétorique : de manière tantôt glaciale, tantôt passionnée, et souvent fallacieuse. La littérature sur le sujet est vertigineuse par son volume et les aspects qu'elle embrasse mais un seul nous intéresse ici. Pour les besoins de la démonstration, les choses paraissent limpides d'évidence : nés pour servir et obéir, les Noirs ont dû désobéir en choisissant la voie héroïque de la révolution pacifiste. Les conséquences sur le droit fédéral furent réelles et les lois, les décisions de justice, ont progressivement épousé les mouvements de la rue en ouvrant aux Noirs la voie de l'émancipation. Mais, sans doute en raison d'une culture d'opposition des races trop ancrée dans cette jeune nation, les faits n'ont pas suivi le droit. Et ce n'est pas l'élection de Barack Obama qui permet d'oublier qu'il est encore, aujourd'hui, plus facile de naître blanc que noir sur le sol américain. Pour que la désobéissance des années 1950 et 1960 porte effectivement ses fruits, pour que l'histoire des États-Unis puisse être enfin racontée autrement qu'à travers la querelle des races, il fallait franchir l'étape de l'intégration. Ce que l'on appelle à tort en France les discriminations positives en faveur des Noirs - en réalité, des actions positives - ne devraient jamais être comparées aux politiques analogues visant à privilégier d'autres catégories anciennement négligées. Non que celles-ci soient moins nobles ou moins urgentes. Mais en raison de la spécificité de la position du Noir démontrée dans les lignes qui précèdent, la politique visant à forcer leur intégration l'est tout autant.

Le second aspect de l'affirmative action qu'il faut ici mettre en lumière est sa dette envers le père de la désobéissance civile des Noirs. C'est à propos de cette ultime étape de l'intégration que Martin 
Luther King a les seuls mots, non haineux, mais fermes et directifs. Il s'adresse aux Blancs en ces termes : «Les Noirs ont soif de justice et pas seulement d'amour. Il ne suffit pas de dire : « Nous aimons les Noirs, nous avons beaucoup d'amis noirs. " Il faut exiger qu'il leur soit fait justice. L'amour qui ne s'acquitte pas de sa dette de justice ne mérite pas son nom. Ce n'est qu'une affection sentimentale, comme celle qu'on accorde à un animal familier. Au meilleur sens du terme, aimer, c'est faire appliquer la justice 38. » Une autre lecture de Martin Luther King permet de le présenter comme l'un des inspirateurs de l'affirmative action. Tous les éléments de cette politique (instaurer des inégalités de droit pour com- penser des inégalités de fait), qu'il qualifie lui-même de « préférentielle et compensatoire 39 », se retrouvent dans son œuvre : "Il ne suffit pas de transformer radicalement son attitude envers les Noirs, sous la pression des événements ; le pays doit aussi envisager des compensations aux handicaps que les Noirs ont subis dans le passé [...], afin de rétablir l'équilibre et leur permettre d'entrer dans la compétition sur des bases justes et équitables 40. » Et Martin Luther King de proposer, pour les Noirs, un équivalent du GI Bill of Rights de 1944 (une politique préférentielle en faveur des anciens combattants). Ce long passage est la meilleure définition qui puisse être donnée des discriminations positives et de leur légitimité.

L'histoire de l'affirmative action aux États-Unis et notamment de son traitement par le pouvoir judiciaire est bien connue 41. Comme le sont les positions de certains juges estimant « suspecte » toute distinction basée sur la race alors même qu'ils ne sont pas vraiment des nigger-lovers 42 . Une manière d'affirmer implicitement que l'on repousse les effets d'une politique préférentielle (l'intégration des Noirs) au nom d'une prétendue posture égalitariste (ne pas stigmatiser une race) 43. C'est si simple - en réalité, si hypocrite - de dire : «C'est parce que je ne suis pas raciste que je ne veux pas que le critère de la race soit utilisé comme critère de distinction. » Or sans ce critère distinctif - lequel, sans être pour autant toujours suspect, est potentiellement dangereux -, les Noirs ne peuvent accéder à la « vraie vie ». Pas celle inscrite dans les supports des beaux idéaux, mais celle de l'emploi, du logement, de la santé et de l'éducation. Et si les Noirs ont désobéi en refusant de céder la place à un Blanc dans un bus ou en buvant de l'eau à une fontaine réservée aux Blancs, ce n'était pas seulement pour que la majorité de la société arrête de les considérer comme des êtres inférieurs ou de les séparer. Ce n'était pas seulement pour survivre et vivre. C'était aussi pour être. Et quand l'égalité ne peut triompher par elle-même, quand les proclamations et condamnations ne suffisent pas à transformer les mentalités, il faut se servir non plus de l'arme massive de la désobéissance pacifiste, mais de l'arme ferme et rigoureuse du droit, dût-elle être utilisée de manière sophistiquée.

\section{Notes de bas de page}

1. William Edward Burghardt Du Bois, Les Âmes du peuple noir (1903), Paris, La Decouverte, 2007, p. 7.

2. Lauren Robel et Elisabeth Zoller, Les États des Noirs, Paris, puf, 2000, p. 1.

3. Martin Luther King, Révolution non violente (1964), Paris, Petite bibliotheque Payot, 2006, p. 172.

4. Et peu importent les discours tendancieux tentant de de-symboliser l'evenement en mettant l'accent sur l'ascendance de Barack Obama, lequel ne serait pas vraiment 《 afro-americain $\gg$. 5. Lord Byron, cite par William Edward Burghardt Du Bois, Les Âmes du peuple noir, op. cit., p. 46.

6. Martin Luther King, Révolution non violente, op. cit., p. 36 (en italiques dans le texte).

7. Martin Luther King, Black Power (1967), Paris, Petite bibliotheque Payot, 2008, p. 126. 
8. Bernard Vincent, Lincoln. L'homme qui sauva les États-Unis, Paris, L'Archipel, 2009, p. 123-124. 9. 41 US 539.

10. 60 US (19 How.) 393.

11. Cite par Donald E. Lively, Foreshadows of the Law: Supreme Court Dissents and Constitutional Development, Westport (Conn.)-Londres, Praeger, 1992, p. 12 (nous soulignons). Sauf mention contraire, les traductions sont de nous.

12. Cite par Elisabeth Zoller, Grands arrêts de la Cour suprême des États-Unis, Paris, puf, 2000, p. 212. 13. Ibid., p. 216 (nous soulignons).

14. Thomas Jefferson, Notes on the State of Virginia (1785), cite par Kermit L. Hall, Paul Finkelman et James W. Ely Jr., American Legal History: Cases and Materials, 3e ed., New York (N. Y.), Oxford University Press, 2005, p. 101.

15. William Edward Burghardt Du Bois, Les Âmes du peuple noir, op. cit., p. 44.

16. 347 US 483.

17. C'est la these de Lauren Robel et Elisabeth Zoller, Les États des Noirs, op. cit., p. 4 : « Aux EtatsUnis, la question raciale ne s'est jamais separee du federalisme et le federalisme n'a jamais pu se detacher de la race. $\gg$

18. Cite par Elisabeth Zoller, Les Grands Arrêts de la Cour suprême des États-Unis, Paris, Dalloz, 2012, p. 287.

19. Ibid., p. 288.

20. Ibid., p. 289.

21. 163 US 537.

22. Elisabeth Zoller, Grands arrêts de la Cour suprême des États-Unis, op. cit., p. 311.

23. Henry David Thoreau, La Désobéissance civile (1849), Paris, Mille et une nuits, 2000, p. 15 (en italiques dans le texte).

24. Ibid., p. 28.

25. Etienne de La Boetie, Discours de la servitude volontaire (1546-1548; date de rédaction presumee selon Montaigne), Paris, Mille et une nuits, 1995, p. 12.

26. Titre de la traduction francaise originale de Black Power, op. cit.

27. William Edward Burghardt Du Bois, Les Âmes du peuple noir, op. cit., p. 62.

28. Martin Luther King, Révolution non violente, op. cit., p. 97 et 30.

29. Le terme est employe par William Edward Burghardt Du Bois, Les Âmes du peuple noir, op. cit., p. 8 $: \ll[\ldots]$ je suis de la chair et du sang de ceux qui vivent dans le voile $\gg$.

30. Martin Luther King, Révolution non violente, op. cit., p. 167 et 69.

31. Ibid., p. 108.

32. Martin Luther King, Black Power, op. cit., p. 152.

33. Ibid., p. 108.

34. Ibid., p. 98 (en italiques dans le texte).

35. David Bradley, L'Incident (1981), Paris, Denoel, 1984, p. 148.

36. William Edward Burghardt Du Bois, Les Âmes du peuple noir, op. cit., p. 107.

37. Pour une presentation generale, cf. Gwenaele Calves, La Discrimination positive, Paris, puf, coll. « Que sais-je ? 》, 2010.

38. Martin Luther King, Black Power, op. cit., p. 106.

39. Martin Luther King, Révolution non violente, op. cit., p. 193.

40. Ibid., p. 192-193.

41. Cf. en particulier Regents of the University of California v. Bakke, 438 US 265 (1978) ; City of

Richmond v. J.A. Croson Co, 488 US 469 (1989); et Gratz v. Bollinger, 539 US 244 (2003). 
42. Litteralement, 《 chouchouteurs de negres $\gg$.

43. Cf. notamment l'opinion separee du juge Scalia sous la decision Croson precitee. 